
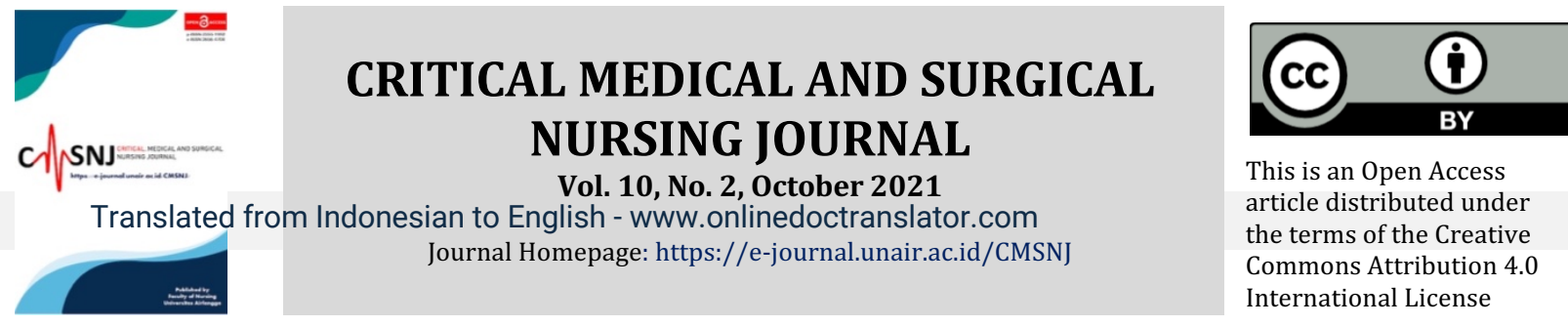

This is an Open Access article distributed under the terms of the Creative Commons Attribution 4.0 International License

\title{
Principles of Providing Nutrition to People Living with HIV/AIDS : A Literature Review
}

\author{
Ninuk Dian Kurniawati ${ }^{1}{ }^{\circledR}$, Adi Sukma Septiana², Muhammad Iqbal ${ }^{2}$, Laely Sholihah², Dyah \\ Sekaringtyas Ciptaningrum ${ }^{2}$, Yulita Thadea Retanubun ${ }^{2}$ \\ ${ }^{1}$ Faculty of Nursing, Airlangga University, Surabaya, Indonesia \\ 2 Bachelor Student, Faculty of Nursing, Universitas Airlangga, Surabaya, Indonesia
}

\begin{abstract}
ARTICLE HISTORY
Received: October 11, 2021

Accepted: December 10, 202

Published: December 10, 2021

\section{KEYWORDS}

ABSTRACT

Introduction: HIV patients with good nutritional status have a good immune system. Good nutritional status can inhibit the process of HIV disease entering the AIDS stage. In addition, good nutritional intake is needed so patients can maintain their health conditions during ARV treatment. This literature review aims to explain the principles of nutritional fulfilment in HIV patients.
\end{abstract}

nutrition, micronutrients, macronutrients, PLWHA, HIV, AIDS

\section{CORRESPONDING AUTHOR}

Ninuk Dian Kurniawati ninuk-dk@fkp.unair.ac.id

Faculty of Nursing, Universitas Airlangga, Surabaya, Indonesia

Cite this as:
Methods: The method used in this study is a literature review with predetermined inclusion and exclusion criteria. The number of articles obtained from the literature review results is 8 articles. The instrument used to evaluate the research is from The Joanna Briggs Institute (JBI).

Results: A total of 8 articles met the inclusion and exclusion criteria. The results of the study assessment from the article achieved a score higher than $50 \%$ so that it met the Critical Appraisal criteria which would then be analyzed for data. Inadequate nutritional status in a person with HIV-AIDS (PLWHA) has a high risk of declining health in general. With good nutritional status, it can prevent HIV disease from entering the AIDS stage.

Conclusion: Protein-energy enriched macronutrient supplementation and ARV initiation effectively improve physiological, nutritional status and immune response in PLWHA. This can prevent people living with HIV from experiencing malnutrition and wasting, restore the ideal body weight, increase and maintain the body's ability to fight various opportunistic infections, increase the effect of drugs, and improve and improve the quality of life of PLWHA patients.

\section{INTRODUCTION}

Adequate nutritional intake plays an important role in supporting health and improving the quality of life of PLWHA(Yuniarti, Purba and Pangastuti, 2018). Nutrition management given to PLWHA patients can help maintain optimal nutritional status of HIV/AIDS patients so that they can help sufferers to carry out regular activities. Knowledge about good nutrition and support from family and community regarding food utensils and good food preparation greatly affect the nutritional status of HIV/AIDS sufferers, how respondents experience changes in their diet since knowing about their health status(Ewune et al., 2021).
Inadequate nutritional status in a person with HIVAIDS (PLWHA) has a high risk of declining health in general. If a person is infected with HIV but has a good nutritional status, his immune system will be better than someone who is infected with HIV but has poor nutritional status. With good nutritional status, it can prevent HIV disease from entering the AIDS stage. Malnutrition that occurs in a person with HIV/AIDS can accelerate the progression of HIV disease and hinder treatment, thereby worsening the patient's condition. Antiretroviral (ARV) use. The condition of PLWHA can decrease if there are effects in the use of ARVs that are not accompanied by good nutritional intake(Yuniarti, Purba and Pangastuti, 2018). 
Malnutrition at the beginning of the use of antiretroviral (ARV) can cause a decrease in the quality of life of PLWHA patients. Thus, there is a need for the introduction of protein-energy-enriched macronutrient supplements in the initiation of ARVs. This study revealed that antiretroviral therapy does affect some serum mineral and vitamin levels and that there are differences based on which drug is used. Thus, the effect of minerals is important on the development and management of HIV AIDS.(Shah et al., 2019). It can improve HIV treatment outcomes and positively affect nutritional status and immunological responses in PLWHA(Hong, Budhathoki and Farley, 2018).

\section{METHOD}

This research design uses a literature review or literature review. The collection of data sources is done by collecting data or sources related to the topic obtained from existing research journals.

\section{Inclusion Criteria}

1. Articles that discuss the principles of providing nutrition to PLWHA

2. No intervention given

3. There is no comparison factor

4. The research design used is a literature review

5. Publication year between $2016-2021$

6. The language used in the article is English and Indonesian

\section{Exclusion Criteria}

1. The research design used is ex post facto comparative, true experiment, quasiexperimental, single subject

2. Incomplete text or full text on the article

3. Articles that do not explain about providing nutrition to PLWHA.

The total number of samples obtained from the results of the literature review is 128 respondents. The instrument used in evaluating the research is using an instrument from The Joanna Briggs Institute (JBI).

\section{RESULT}

The results of the study assessment from the article achieved a score higher than $50 \%$ so that it met the Critical Appraisal criteria for which data analysis would then be carried out. Inadequate nutritional status in a person with HIV-AIDS (PLWHA) has a high risk of declining health in general. With good

62 | Volume 10 No. 2 OCTOBER 2021 nutritional status, it can prevent HIV disease from entering the AIDS stage.

\section{DISCUSSION}

The principles of good nutrition in helping to maintain the ideal body weight of people with HIV/AIDS are by: preventing malnutrition and wasting, providing strong immunity and resistance, reducing disease and slowing the progression of HIV/AIDS, achieving a better life and getting a better quality of life. good. Here are some steps to get good nutrition:

a. Pay attention to diet and discuss diet and related issues with your doctor or nutritionist.

b. Regular exercise can help relieve stress and stimulate appetite.

c. Drink at least 8 glasses of water a day

d. Avoid alcohol

e. Consume vitamins and minerals

1. Vitamin A such as: fruits, spinach, broccoli, sweet potatoes, green peppers, pumpkin, carrots, liver, butter, cheese, eggs)

2. B vitamins such as: white beans, beans, avocados, broccoli, and green leafy vegetables.

3. Vitamin C such as: oranges, grapes, lemons, guavas, mangoes, tomatoes, and potatoes.

4. Vitamin E such as: green leafy vegetables, vegetable oils, peanuts and egg yolks (Nadhiroh, 2016).

f. Vit. D increasing sun exposure to the body, taking supplements or foods that contain vit. D, all of these things must be adapted to the needs of each individual's body (Mumena et al., 2021).

g. Zinc and minerals can help activate the immune system, such as: whole grains, whole wheat bread, corn and milk.

h. Iron such as: red meat, seeds, liver, chicken, nuts, milk, peanuts, etc.

Several people living with HIV in this study experienced anorexia (47\%) and nausea (47\%). Loss of appetite occurs due to an increase in interleukin-1, interleukin-6, tumor necrosis factor, gastrointestinal infections, advanced manifestations of HIV disease (fatigue, fever, and dyspnea), disabilities that interfere with a person living with HIV both to eat and get food. Indicators in measuring nutritional status can use changes in body weight and BMI which are categorized into 2, namely BMI < 18.5 (malnutrition status), BMI = 18.5-22.9 (normal nutritional status), and BMI > 22.9 (normal nutritional status). over nutrition). Nutrition counseling for PLWHA is very much needed, because counseling can provide a good influence on the development of nutritional intake for 
PLWHA. Counseling is provided in the form of information about food ingredients that are recommended and not recommended for consumption,(Yuniarti, Purba and Pangastuti, 2018).

The process of nutrition counseling care for PLWHA includes:

a. Nutritional Assessment

1. Anthropometric measurements and body composition, assessing whether the PLWHA patient is experiencing malnutrition or excess nutrition.

2. Wasting and lipodystrophy for measuring the circumference of the thigh, forearm, and upper middle arm.

3. Overweight, monitoring of changes in body composition is recommended during a planned weight loss program.

4. Measurement of body composition. BMI can be used to assess height and weight regarding general body measurements.

5. Biochemical assessment: measurement of serum protein, lipid, glucose, and micronutrients.

6. Anemia, ferritin level $<200 \mathrm{ng} / \mathrm{L}$ or transferrin saturation $<20 \%$ may indicate iron deficiency

7. Lipids and blood pressure

8. Diabetes, checking insulin levels, glucose, screening people living with HIV for diabetes and prediabetes at 6 to 12 month intervals before starting ART

9. Hormones, hormone levels can affect nutritional status and the risk of chronic disease in PLWHA.

10. Dietary assessment, identification of factors that influence food intake

11. ART assessment, nutrition and nutritional status can affect the absorption, utilization, elimination, and tolerance of ART drugs.

b. Intervention

1. The MNT value provided by the RDN has a significant value and is recommended for all people with HIV infection

2. Client education and counselling, nutrition education and counseling can have a positive effect on health outcomes.
3. Macronutrient, absolute resting energy expenditure (REE) adjusted for fat-free mass (REE/FFM).

4. Micronutrients, vitamin D and calcium supplementation may reduce the loss of bone density observed with ART.

c. Comorbid $=$ there are several comorbidities including osteoporosis, CVD, hypertension, diabetes, and infection.

d. Monitoring and evaluation that can be done are:

1. Food and drink intake and availability, CAM use, and food allergies

2. Anthropometric measurement of outcomes including body weight and body composition

3. Results of biochemical data including HIV viral load, serum lipids, and glucose levels

4. Physical findings that are nutritionally focused outcomes include fat and muscle loss(Willig, Wright and Galvin, 2018).

Nutrition counseling for PLWHA is very much needed, because counseling can provide a good influence on the development of nutritional intake for PLWHA. The counseling provided is in the form of information about food ingredients that are recommended and not recommended for consumption, diet strategies according to the patient's condition, dietary requirements, as well as providing tips to patients who have problems with their health status. Nutrition counseling can increase food intake in PLWHA. This can prevent people living with HIV from experiencing malnutrition and wasting, can restore the ideal body weight, increase and maintain the body's ability to fight various opportunistic infections, increase the effect of drugs, and can improve and improve the quality of life of patients living with HIV.(Yuniarti, Purba and Pangastuti, 2018)

\section{CONCLUSION}

Protein-energy enriched macronutrient supplementation along with ARV initiation is effective in improving physiological nutritional status and immune response in PLWHA. This can prevent PLWHA from experiencing malnutrition and wasting, can restore the ideal body weight, increase and maintain the body's ability to fight various

http://e-journal.unair.ac.id/CMSNJ | 63 
opportunistic infections, increase the effect of drugs, and can improve and improve the quality of life of PLWHA patients.

\section{REFERENCE}

Ewune, HA et al. (2021) 'Challenges to nutrition management among patients using antiretroviral therapy in primary health "centres" in Addis Ababa, Ethiopia: A phenomenological study', PLoS ONE, 16(6 June), pp. 1-15. doi: 10.1371/journal.pone.0250919.

Hong, H., Budhathoki, C. and Farley, JE (2018) 'Effectiveness of macronutrient supplementation on nutritional status and HIV/AIDS progression: A systematic review and meta-analysis', Clinical Nutrition ESPEN. Elsevier Ltd, 27, pp. 66-74. doi: 10.1016/j.clnesp.2018.06.007.

Morvaridzadeh, M. et al. (2020) 'The effects of omega3 fatty acid supplementation on inflammatory factors in HIV-infected patients: A systematic review and meta-analysis of randomized clinical trials', Cytokines. Elsevier, 136(August), p. 155298. doi: 10.1016/j.cyto.2020.155298.

Mumena, CH et al. (2021) 'The relevance of vitamin D in the oral health of HIV infected patients', Journal of Steroid Biochemistry and Molecular Biology. Elsevier Ltd, 211(April), p. 105905. doi:10.1016/j.jsbmb.2021.105905.

Nadhiroh, SR (2016) 'Good Nutrition for Quality of Life of PLWHA (People Living with HIV/AIDS)', pp. 29-34.

Shah, KK et al. (2019) 'Essential trace elements and progression and management of HIV infection', Nutrition Research. Elsevier Inc., 71, pp. 21-29. doi: 10.1016/j.nutres.2019.08.001.

Willig, A., Wright, L. and Galvin, TA (2018) 'Practice Paper of the Academy of Nutrition and Dietetics: Nutrition Intervention and Human Immunodeficiency Virus Infection', Journal of the Academy of Nutrition and Dietetics, 118(3), pp. 486-498. doi: 10.1016/j.jand.2017.12.007.

Yuniarti, Y., Purba, MB and Pangastuti, R. (2018) 'The effect of nutritional counseling and food supplementation on nutritional intake and nutritional status of HIV/AIDS patients', Indonesian Journal of Clinical Nutrition, 9(3), p. 132. doi:10.22146/ijcn.15446.

64 | Volume 10 No. 2 OCTOBER 2021 